OPEN ACCESS

Edited by:

Yuxin Tang,

University of Macau, China

Reviewed by:

Xihong Lu,

Sun Yat-Sen University, China

Shengjie Peng,

Naniing University of Aeronautics and

Astronautics, China

Xingtao $\mathrm{Xu}$,

Hohai University, China

*Correspondence:

Jiangnan Shen

shenjn@zjut.edu.cn

Xiehong Cao

gcscaoxh@zjut.edu.cn

${ }^{\dagger}$ These authors have contributed equally to this work

Specialty section:

This article was submitted to

Electrochemistry,

a section of the journal

Frontiers in Chemistry

Received: 03 May 2019

Accepted: 04 June 2019

Published: 19 June 2019

Citation:

Shi W, XuX, Ye C, Sha D, Yin $R$

Shen X, Liu X, Liu W, Shen J, Cao X

and Gao C (2019) Bimetallic

Metal-Organic Framework-Derived

Carbon Nanotube-Based Frameworks for Enhanced Capacitive Deionization

and $\mathrm{Zn}$-Air Battery.

Front. Chem. 7:449.

doi: 10.3389/fchem.2019.00449

\section{Bimetallic Metal-Organic Framework-Derived Carbon Nanotube-Based Frameworks for Enhanced Capacitive Deionization and Zn-Air Battery}

\author{
Wenhui Shi ${ }^{1+}$, Xilian $X u^{2+}$, Chenzeng $\mathrm{Ye}^{1+}$, Dongyong Sha ${ }^{2}$, Ruilian Yin ${ }^{2}$, Xuhai Shen ${ }^{2}$, \\ Xiaoyue $\mathrm{Liu}^{1}$, Wenxian $\mathrm{Liu}^{2}$, Jiangnan Shen ${ }^{1 *}$, Xiehong $\mathrm{CaO}^{2 \star}$ and Congjie Gao ${ }^{1}$ \\ ${ }^{1}$ Center for Membrane Separation and Water Science and Technology, Ocean College, Zhejiang University of Technology, \\ Hangzhou, China, ${ }^{2}$ College of Materials Science and Engineering, Zhejiang University of Technology, Hangzhou, China
}

Carbon-based materials have attracted intensive attentions for a wide range of energy and environment-related applications. Energy storage/conversion devices with improved performance have been achieved by utilization of metal-organic-framework (MOF)-derived carbon structures as active materials in recent years. However, the effects of MOF precursors on the performance of derived carbon materials are rarely investigated. Here, we report that the incorporation of small amount of $\mathrm{Fe}$ or $\mathrm{Ni}$ in Co-based MOFs leads to a significant enhancement for the derived carbon nanotube-based frameworks (CNTFs) in $\mathrm{Na}^{+} / \mathrm{Cl}^{-}$ion electrosorption. Further investigation revealed the enhanced performance can be attributed to the improved specific surface area, electrical conductivity, and electrochemical activity. Notably, the CoFe-CNTF derived from bimetallic CoFe-MOFs achieves a high ion adsorption capacity of $37.0 \mathrm{mg} \mathrm{g}^{-1}$, superior to most of recently reported carbon-based materials. Furthermore, the CoFe-CNTF also demonstrates high catalytic activity toward oxygen evolution reaction (OER) with a Tafel slope of $87.7 \mathrm{mV} \mathrm{dec}^{-1}$. After combination with three-dimensional graphene foam (3DG), the resultant CoFe-CNTF-coated 3DG is used as air-cathode to fabricate a flexible all-solid-state Zn-air battery, which exhibits a high open circuit potential of $1.455 \mathrm{~V}$. Importantly, the fabricated flexible battery can light a light-emitting diode (LED) even when it is bent. This work provides new insights into designs of high-performance and flexible electrode based on MOF-derived materials.

Keywords: metal-organic frameworks, carbon nanotubes, capacitive deionization, oxygen evolution reaction, $\mathrm{Zn}$-air battery, flexible devices, three-dimensional graphene, hierarchical structures

\section{INTRODUCTION}

Metal-organic frameworks (MOFs), as a group of porous crystalline materials with tailorable composition and structure, are versatile precursors for carbon-based porous materials (Tang et al., 2015; Xu et al., 2017, 2018). MOF-derived carbon-based materials have attracted intensive attentions for a wide range of applications, benefiting from their high surface area and well-defined porosity (Wang et al., 2016, 2017b, 2019; Li et al., 2018b; Liu et al., 2019a). Capacitive deionization 
(CDI) has emerged as a potential water treatment technology owing to its low cost and energy consumption (Song et al., 2019; Xu et al., 2019). In the CDI process, upon the application of an external voltage, ions in the solution are adsorbed and stored in the electrodes by forming electrical double layers (Suss et al., 2015; Hassanvand et al., 2018). A series of carbon-based materials have been extensively studied as CDI electrodes, such as activated carbon (Wu et al., 2016; Tang et al., 2019), graphene (Cao et al., 2014; Shi et al., 2016; Liu et al., 2017a; Li et al., 2018a), mesoporous carbon (Tsouris et al., 2011; Wang et al., 2014; Gao et al., 2019b), carbon nanotubes (Nie et al., 2012; Liu et al., 2015) and their composites (Xu et al., 2016b; Huang et al., 2017; Hu et al., 2018). Recently, MOF-derived porous carbon has demonstrated great potential for CDI applications. However, MOF-derived carbon materials generally have poor electrical conductivity because of low crystallinity of carbon (Gao et al., 2018, 2019a). Furthermore, abundant micropores within MOF-derived carbon structures limit the diffusion of ions into the electrode, leading to unsatisfactory CDI performance (Ding et al., 2017; Shi et al., 2018, 2019a). Therefore, novel strategies are required to further improve electron and ion transport of MOF-derived carbons (Wang et al., 2017a; Shi et al., 2019b).

In addition, MOF-derived nanostructured carbons are also attractive noble-metal-free electrocatalysts, which are promising in oxygen evolution reaction (OER) and have been used as cathode materials for Zn-air batteries recently (Liu et al., 2018, 2019b; Zhang et al., 2018b; Fang et al., 2019; Guo et al., 2019). Although the bright prospects of MOF-derived carbon materials, their practical applications are still restricted. First, micropores within MOF-derived porous carbon materials contribute to the majority of specific surface area, which have limited diffusion rate for electrolyte ions. Second, the development of nextgeneration flexible energy storage/conversion devices requires electrode materials with robust structure and strong mechanical properties under deformation (Han et al., 2018; Lv et al., 2018; Peng et al., 2018; Zhu et al., 2018; Ji et al., 2019). However, MOFderived carbons are normally in powder form, which is difficult to form a firm interaction within a flexible electrode. Third, previous reports have shown that metallic content within MOFderived carbons play an essential role in their catalytic properties (Liu et al., 2017b; Zhang et al., 2018a; Zheng et al., 2019). The effects of bimetallic MOF precursors on the performance of derived carbon materials are rarely investigated. Therefore, it is still a challenge to develop hierarchically porous, flexible, highion/charge-transport-rate MOF-derived carbon-based materials for high-performance $\mathrm{Zn}$-air battery.

In this work, unique carbon nanotube-based frameworks (CNTFs) were obtained by a facile annealing process of rationally designed bimetallic MOF crystals (i.e., CoFe and CoNi-MOFs) as precursors. By the incorporation of a small amount of $\mathrm{Fe}$ or $\mathrm{Ni}$ in Co-based frameworks, the obtained CoFe- and CoNi-CNTFs both exhibited improved specific surface area and electrical conductivity, as compared to those of Co-CNTF. Due to the hierarchically porous structure with abundant exposed active sites, high conductivity for fast electron transfer, and unique nanostructures with metal nanoparticle embedded in CNTs, the $\mathrm{CoFe}$ - and CoNi-CNTF electrodes exhibited remarkably enhanced $\mathrm{Na}^{+} / \mathrm{Cl}^{-}$ions adsorption property. Furthermore, high catalytic activity toward oxygen evolution reaction (OER) was also achieved by CoFe-CNTF. As a proof-of-concept application, an air-cathode based on CoFe-CNTF coated 3D graphene foam (CoFe-CNTF@3DG) was fabricated, and then used for flexible all-solid-state $\mathrm{Zn}$-air battery, which displayed a high open circuit potential of $1.455 \mathrm{~V}$.

\section{EXPERIMENTAL SECTION}

\section{Material Synthesis}

Preparation of Co-MOF: Typically, $1.4553 \mathrm{~g}$ of $\mathrm{Co}\left(\mathrm{NO}_{3}\right)_{2} \cdot 6 \mathrm{H}_{2} \mathrm{O}$ was dissolved in $50 \mathrm{~mL}$ of methanol, which was mixed with $50 \mathrm{~mL}$ of methanol containing $3.2860 \mathrm{~g}$ of 2 -methylimidazole. The obtained mixture was stirred at room temperature for $12 \mathrm{~h}$. The sample was collected by centrifugation and washed with methanol for several times, followed by drying at $60^{\circ} \mathrm{C}$ for $12 \mathrm{~h}$ in vacuum oven.

Preparation of bimetallic CoFe and CoNi-MOFs: CoFe-MOF was synthesized by using $\mathrm{Co}\left(\mathrm{NO}_{3}\right)_{2} \cdot 6 \mathrm{H}_{2} \mathrm{O}$ and $\mathrm{Fe}\left(\mathrm{NO}_{3}\right)_{3} \cdot 6 \mathrm{H}_{2} \mathrm{O}$ with a molar ratio of 9:1. Typically, $1.3097 \mathrm{~g}$ of $\mathrm{Co}\left(\mathrm{NO}_{3}\right)_{2} \cdot 6 \mathrm{H}_{2} \mathrm{O}$ and $0.2019 \mathrm{~g}$ of $\mathrm{Fe}\left(\mathrm{NO}_{3}\right)_{3} \cdot 6 \mathrm{H}_{2} \mathrm{O}$ were dissolved in $50 \mathrm{~mL}$ of methanol. Then, $50 \mathrm{~mL}$ of methanol dissolved with $3.2860 \mathrm{~g}$ of $2-$ methylimidazole was added into the above solution followed by continuously stirring for $12 \mathrm{~h}$ at room temperature. CoFe-MOF was collected by centrifugation and washed with methanol for several times, followed by drying at $60^{\circ} \mathrm{C}$ for $12 \mathrm{~h}$ in vacuum oven. CoNi-MOF was synthesized by a similar procedure using $\mathrm{Co}\left(\mathrm{NO}_{3}\right)_{2} \cdot 6 \mathrm{H}_{2} \mathrm{O}$ and $\mathrm{Ni}\left(\mathrm{NO}_{3}\right)_{2} \cdot 6 \mathrm{H}_{2} \mathrm{O}$ with a molar ratio of 9:1.

Preparation of CNTFs: The as-prepared MOF precursors were pyrolyzed in a tube furnace by a two-step annealing process. The MOF precursors were first heated to $500^{\circ} \mathrm{C}$ with a heating rate of $5^{\circ} \mathrm{C} \min ^{-1}$, followed by bubbling ethanol vapor using $\mathrm{H}_{2}$ for $30 \mathrm{~min}$. Then, the samples were heated at $800^{\circ} \mathrm{C}$ for $1 \mathrm{~h}$ under $\mathrm{N}_{2}$ atmosphere, followed by immersing the sample in $1 \mathrm{M} \mathrm{HCl}$ for $12 \mathrm{~h}$ to obtain CNTFs.

Preparation of CoFe-CNTF@3DG: 3D graphene foam (3DG) was obtained by using our previously reported method (Cao et al., 2011). Then $3 \mathrm{DG}$ was treated with a concentrated $\mathrm{HNO}_{3}$ solution at $80^{\circ} \mathrm{C}$ for $4 \mathrm{~h}$, followed by washing with deionized water to obtain hydrophilic 3DG. The 3DG was placed into the precursor solution for CoFe-MOF and reacted for $12 \mathrm{~h}$ at room temperature. The CoFe-MOF@3DG composite was obtained by washing with methanol and then drying at $60^{\circ} \mathrm{C}$ for $12 \mathrm{~h}$. The CoFe-CNTF@3DG was obtained according to the aforementioned annealing procedures for CNTF samples.

\section{Material Characterization}

The morphologies of the samples were investigated by fieldemission scanning electron microscopy (FE-SEM, Hitachi SU8010), equipped with energy-dispersive X-ray spectroscopy (EDX) and transmission electron microscopy (TEM, JEM-100CX II). The crystal structures were characterized by powder Xray diffraction (XRD, PNAlytical X'Pert PRO) with $\mathrm{Cu} \mathrm{K \alpha}$ radiation. Nitrogen adsorption/desorption measurements were carried out by Micromeritics ASAP 2020. The specific surface 
areas were calculated by the multipoint Brunauer-Emmett-Teller (BET) method.

\section{Electrosorption Measurements}

Batch-mode CDI tests were conducted by a circulating system, which includes a CDI module, a peristaltic pump (Longer Pump, YZ-1515x), a conductivity meter (Leici, DDSJ-308F) and a source meter (Keithley, SMU-2,400) (Figure S1A). The CDI electrodes were fabricated by coating a mixture of active materials, carbon black and poly(vinylidene fluoride) (PVDF) with a mass ratio of $8: 1: 1$ in $n$-methylpyrrolidone (NMP) solution onto graphite paper, followed by drying at $60^{\circ} \mathrm{C}$ for $12 \mathrm{~h}$ in vacuum oven. As shown in Figure S1B, the CDI module was assembled with a CDI electrode, cation exchange membrane (Hangzhou Grion Environmental Technology Co., Ltd., LEHeCM-1, Type 1), spacer, anion exchange membrane (Hangzhou Grion Environmental Technology Co., Ltd., LEHeAM-I, Type 1) and another CDI electrode. The total volume of $\mathrm{NaCl}$ aqueous solution is maintained at $50 \mathrm{~mL}$. The electrosorption performance was measured at a flow rate of $30 \mathrm{~mL} \mathrm{~min}{ }^{-1}$, varied applied voltages (1.0-1.6 V) and concentrations of $\mathrm{NaCl}$ solution $\left(125-1,000 \mathrm{mg} \mathrm{L}^{-1}\right)$. The electrosorption capacity, $\Gamma\left(\mathrm{mg} \mathrm{g}^{-1}\right)$, is calculated based on the following equation:

$$
\Gamma=\frac{\left(\mathrm{C}_{0}-\mathrm{C}_{\mathrm{t}}\right) V}{\mathrm{~m}}
$$

where $\mathrm{C}_{0}$ is the initial concentration of $\mathrm{NaCl}$ solution $\left(\mathrm{mg} \mathrm{L}^{-1}\right)$, $\mathrm{C}_{\mathrm{t}}$ is the final concentration $\left(\mathrm{mg} \mathrm{L}^{-1}\right), \mathrm{V}$ is the total volume of the solution $(\mathrm{L})$, and $\mathrm{m}$ is the total mass of the electrodes $(\mathrm{g})$.

\section{Electrochemical Measurements}

The electrochemical properties were measured by an electrochemical workstation (Chenhua, China, CHI760E) in a three-electrode system at room temperature, using a carbon paper coated with prepared catalyst as the working electrode, a graphite rod as the counter electrode, a Saturated Calomel Electrode (SCE) as the reference electrode and $1.0 \mathrm{M} \mathrm{KOH}$ as the electrolyte. The catalyst slurry was obtained by adding $5 \mathrm{mg}$ of CoFe-CNTF powder into a mixed solution of $768 \mu \mathrm{L}$ DI water, $200 \mu \mathrm{L}$ ethanol and $32 \mu \mathrm{L}$ nafion, followed by ultrasonication for $30 \mathrm{~min}$. The electrode was fabricated by coating the obtained slurry onto carbon paper with a loading mass of $1 \mathrm{mg} \mathrm{cm}^{-2}$. The Linear Sweep Voltammetry (LSV) was tested at a scan rate of $5 \mathrm{mV} \mathrm{s}^{-1}$ with a potential range from 1.2 to $1.7 \mathrm{~V}$ vs. Reversible Hydrogen Electrode (RHE). The Electrochemical Impedance Spectroscopy (EIS) measurement was conducted by an electrochemical station (Autolab PGSTAT302N) at a potential of $1.33 \mathrm{~V}$ vs. RHE in a frequency range from $100 \mathrm{kHz}$ to $0.01 \mathrm{~Hz}$ with an amplitude of $5 \mathrm{mV}$.

\section{Fabrication of Zn-Air Battery}

$\mathrm{Zn}$-air battery was assembled using a carbon paper loaded with $\mathrm{CoFe}-\mathrm{CNTF}$ catalyst as the air cathode, $\mathrm{Zn}$ plate as the anode, and a mixed solution of $6 \mathrm{M} \mathrm{KOH}$ and $0.2 \mathrm{M} \mathrm{Zn}(\mathrm{Ac})_{2}$ as the electrolyte, which was tested on a battery testing system (Land
CT2001A). The flexible solid-state Zn-air battery was assembled by using CoFe-CNTF@3DG as the air electrode, nickel foam as the current collector, $\mathrm{Zn}$ plate as the anode, and polyacrylic acid (PAA) gel containing $11.25 \mathrm{M} \mathrm{KOH}$ and $0.25 \mathrm{M} \mathrm{ZnO}$ as the solid electrolyte.

\section{RESULTS AND DISCUSSION}

The preparation of CNTF is schematically shown in Scheme $\mathbf{1}$. Bimetallic MOF crystals (i.e., CoFe- and CoNi-MOFs) were first synthesized using the mixtures of metal salts with a proper molar ratio (route 1, see Experimental Section). For comparison, monometallic Co-MOF was also prepared. Then, the as-prepared MOF precursors were undergone a two-step annealing process for pyrolysis of MOFs and catalytic growth of CNTs, followed by an acid treatment to obtain the CNTbased frameworks (i.e., CoFe-CNTF, CoNi-CNTF, and CoCNTF). Furthermore, CoFe-MOFs were in-situ grown on 3D graphene foam (3DG) followed by similar annealing process to produce a flexible air cathode (CoFe-CNTF@3DG, route 2, see Experimental Section).

The scanning electron microscopy (SEM) images of prepared bimetallic MOF crystals exhibit typical dodecahedron shape (Figures S2A-C), which is similar with that of Co-MOF. In addition, the energy-dispersive X-ray spectroscopy (EDX) spectra in Figures S2D-F indicate all of the MOFs show signals of $\mathrm{C}, \mathrm{N}, \mathrm{O}$, and Co. Besides, CoFe- and CoNiMOFs contain additional $\mathrm{Fe}$ or $\mathrm{Ni}$ elements, suggesting the successful incorporation of $\mathrm{Fe} / \mathrm{Ni}$ in the Co-based frameworks. The X-ray diffraction (XRD) patterns in Figure S3 show the same diffraction peaks for $\mathrm{CoFe}-$, CoNi-MOFs, and CoMOF, demonstrating the same crystal structure (Kaur et al., 2016). SEM images in Figure $\mathbf{S} 4$ further reveal that CNTFs derived from $\mathrm{CoFe}, \mathrm{CoNi}$, and Co-MOFs maintained a similar dodecahedron shape after the annealing process, which consist of interconnected CNT frameworks. Further investigations on the morphology and microstructure of CNTFs were performed by transmission electron microscopy (TEM). As shown in Figures 1A-C, the obtained CNTFs exhibited hierarchically porous structures assembled by CNTs, which have nanoparticles with diameters of $10-18 \mathrm{~nm}$ encapsulated on the top of tubes (Li et al., 2016). XRD patterns of all CNTFs exhibit similar characteristic peaks located at $\sim 26$ and $44^{\circ}$ (Figure 1D), which can be assigned to the (002) and (101) planes of graphitic carbon (JCPDS: 41-1487) (Xu et al., 2016a).

The electrosorption performance of the prepared three CNTFs samples for $\mathrm{Na}^{+} / \mathrm{Cl}^{-}$ions were evaluated by measuring change of solution conductivity at applied voltages using a CDI device (Figure S1, see experimental section). As displayed in Figure 1E, when an external voltage was applied, $\mathrm{Na}^{+} / \mathrm{Cl}^{-}$ions were adsorbed and trapped in the electrodes, along with the gradually decreased solution conductivity. The $\mathrm{NaCl}$ electrosorption capacity of the CoFeCNTF electrode is $37.0 \mathrm{mg} \mathrm{g}^{-1}$, which is much higher than that of Co-CNTF $\left(19.9 \mathrm{mg} \mathrm{g}^{-1}\right)$. Figure 1F depicts CDI Ragone plot of the three electrodes, in which the adsorption 


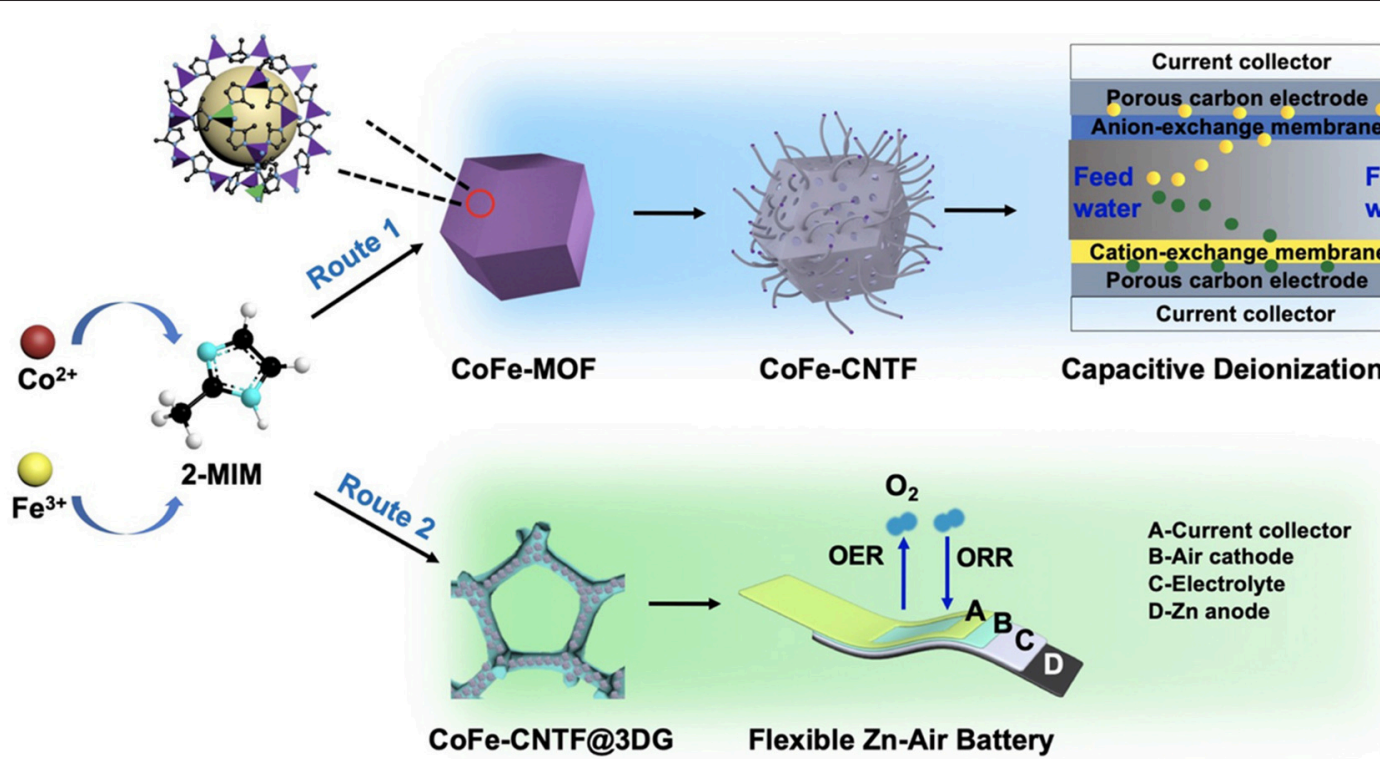

SCHEME 1 | Schematic illustration of the synthetic processes of CoFe-CNTF and CoFe-CNTF@3DG as well as their applications for CDI and Zn-air battery.

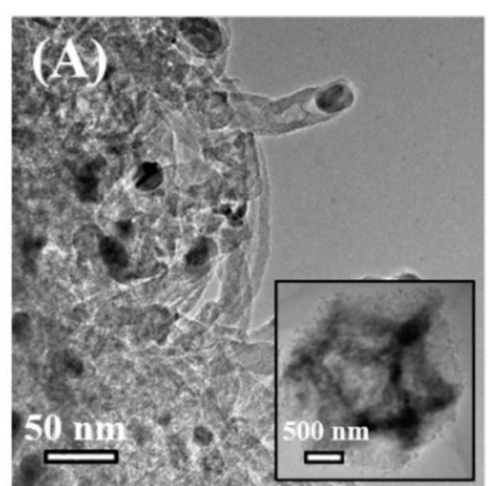

(D)

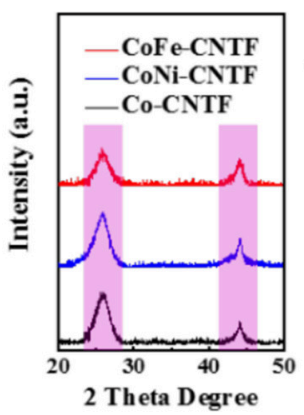

(E)

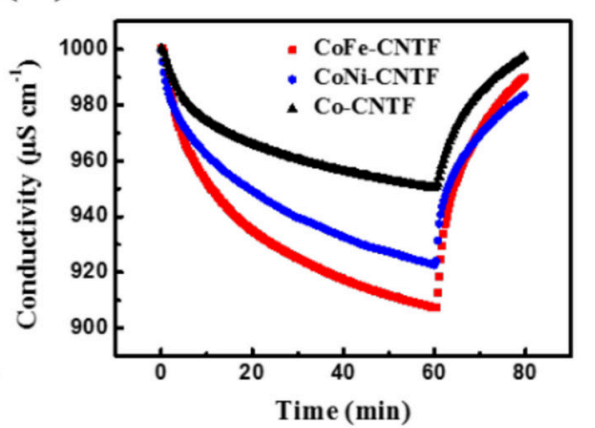

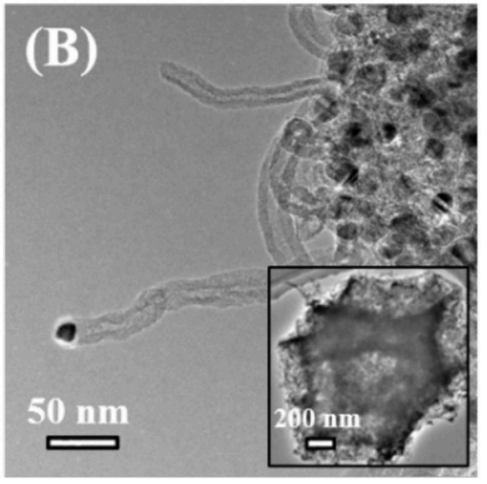

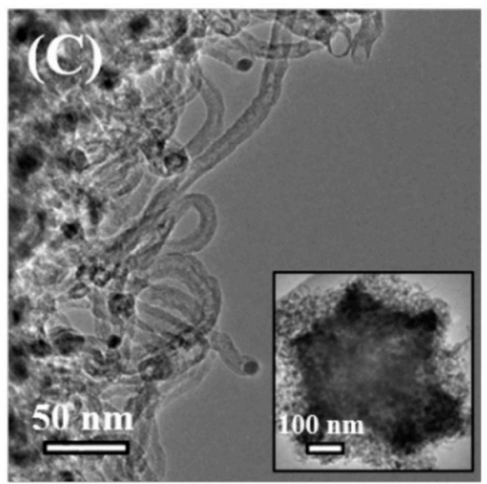

(F)

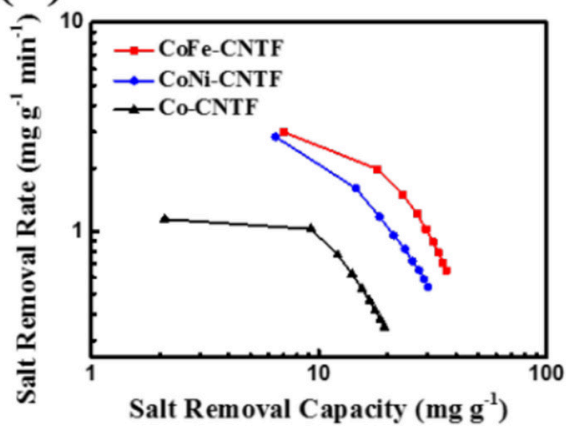

FIGURE 1 | TEM images of (A) CoFe-CNTF, (B) CoNi-CNTF, and (C) Co-CNTF. Insets in (A-C): corresponding low-magnification TEM images. (D) XRD patterns of CoFe-CNTF, CoNi-CNTF, and Co-CNTF. (E) Electrosorption behaviors and (F) CDI Ragone plots of CoFe-CNTF, CoNi-CNTF, and Co-CNTF in 500 mg L ${ }^{-1}$ NaCl solution at $1.2 \mathrm{~V}$.

capacity and rate for $\mathrm{Na}^{+} / \mathrm{Cl}^{-}$ions are used to assess the electrosorption behavior of the electrodes. Notably, in the CDI Ragone plot, the curve of CoFe-CNTF electrode is located in the upper right corner, suggesting both the highest $\mathrm{Na}^{+} / \mathrm{Cl}^{-}$ions adsorption capacity and adsorption rate (Lee et al., 2018). 

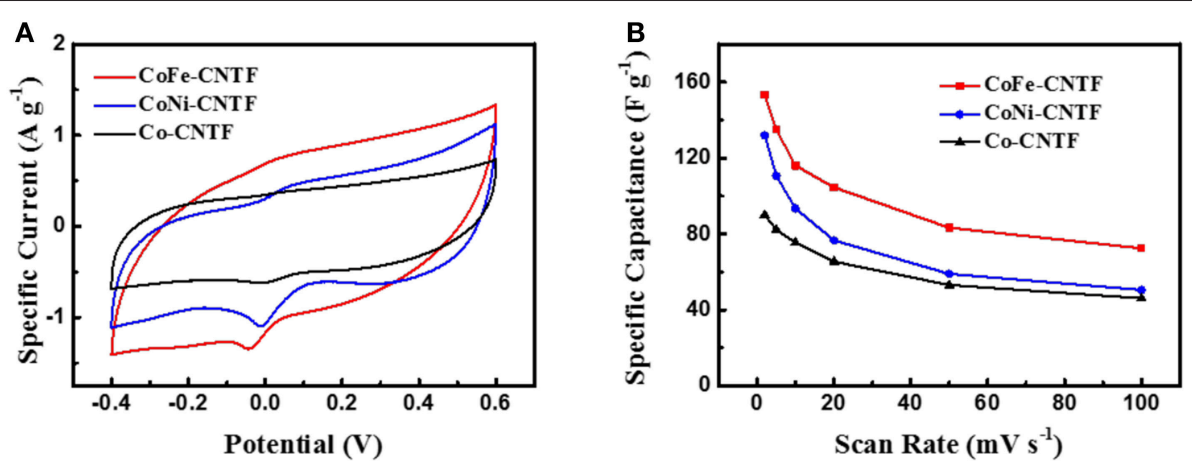

C
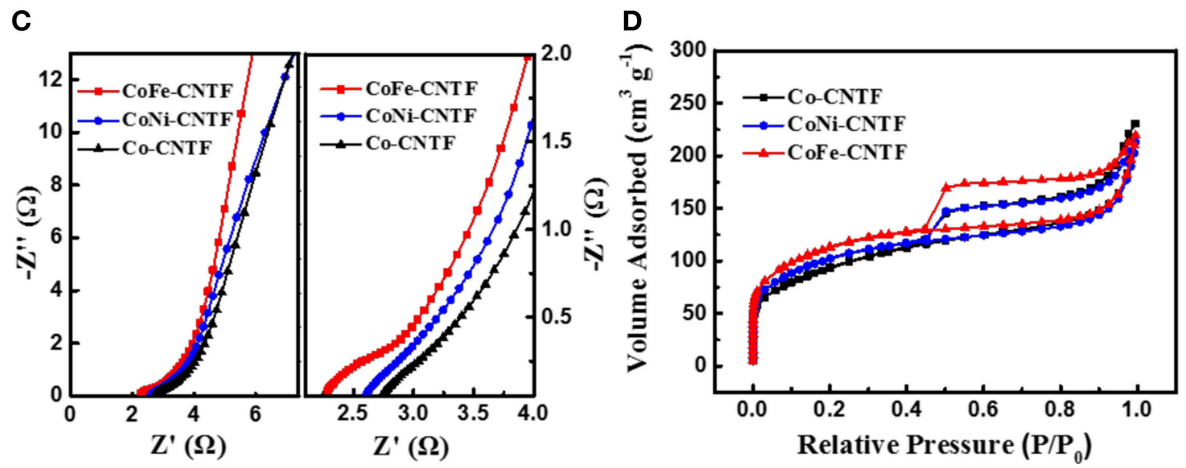

FIGURE 2 | (A) CV curves of CoFe-CNTF, CoNi-CNTF, and Co-CNTF electrodes at a scan rate of $5 \mathrm{mV} \mathrm{s}^{-1}$. (B) Specific capacitances of CoFe-CNTF, CoNi-CNTF, and Co-CNTF at different scan rates. (C) Nyquist plots and (D) Nitrogen adsorption-desorption isotherms of CoFe-CNTF, CoNi-CNTF, and Co-CNTF.
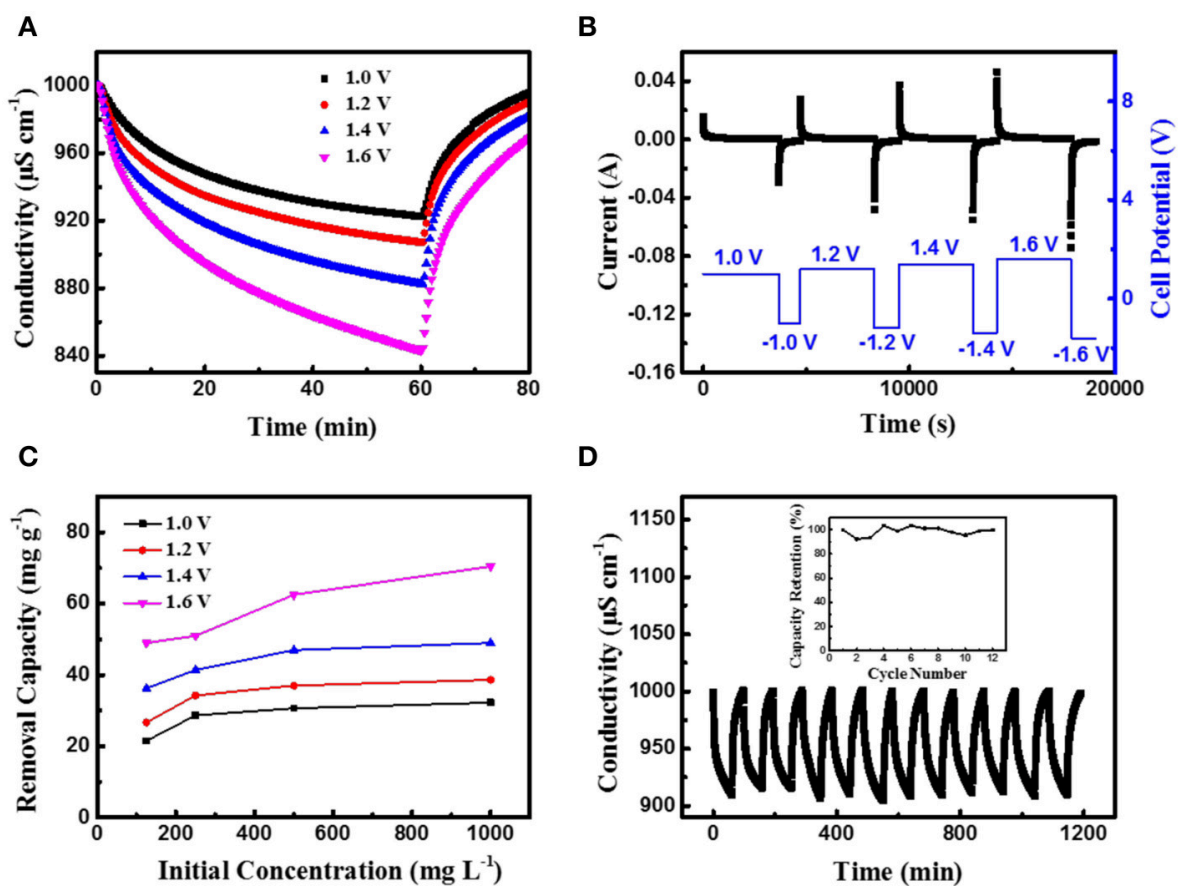

D

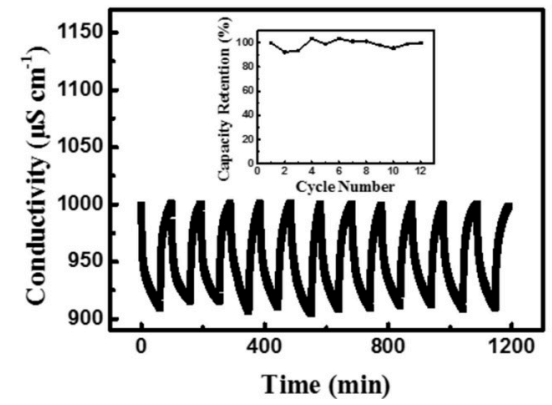

FIGURE 3 | (A) Electrosorption behaviors and (B) the corresponding current curves of CoFe-CNTF in $500 \mathrm{mg} \mathrm{L}^{-1} \mathrm{NaCl}$ solution at various cell voltages. (C) The electrosorption capacity of CoFe-CNTF at various cell voltages and concentrations of $\mathrm{NaCl}$ solution. (D) Cycling performance of $\mathrm{CoFe}-\mathrm{CNTF}$ in $500 \mathrm{mg} \mathrm{L}{ }^{-1} \mathrm{NaCl}$ solution at $1.2 \mathrm{~V}$. Inset is the capacity retention of CoFe-CNTF. 
The aforementioned results demonstrate that the remarkably enhanced $\mathrm{Na}^{+} / \mathrm{Cl}^{-}$ions adsorption performance was achieved by the bimetallic MOF-derived CNTFs. To further reveal the origins of the improvement of CDI performance, further investigations on the properties of samples in terms of electrochemical capacitance, electrical conductivity, and specific surface area were carried out. The cyclic voltammetry (CV) profiles of $\mathrm{CoFe}-$, $\mathrm{CoNi}$-, and $\mathrm{Co}-\mathrm{CNTF}$ electrodes are shown in Figure 2A. Obviously, the CoFe-CNTF electrode exhibited the largest integrated area of $\mathrm{CV}$ curve, which is consistent with the calculated specific capacitances. The CoFe-CNTF electrode showed the highest specific capacitance at various scan rates, i.e., $153 \mathrm{~F} \mathrm{~g}^{-1}$ at $2 \mathrm{mV} \mathrm{s}^{-1}$ and $72 \mathrm{~F} \mathrm{~g}^{-1}$ at $100 \mathrm{mV} \mathrm{s}^{-1}$ (see Figure 2B). To further study the electrical conductivity and internal resistivity of CNTF electrodes, electrochemical impedance spectroscopy (EIS) measurement was conducted. Figure 2C exhibits the Nyquist plots of $\mathrm{CoFe}-, \mathrm{CoNi}-$, and Co-CNTF electrodes in $1 \mathrm{M} \mathrm{NaCl}$ solution, which include a semicircle in the high frequency region and an inclined line in the low frequency region. The inclined line of the CoFe-CNTF electrode shows the largest slope, suggesting faster ion diffusion into the porous structure. In addition, the intercept of the curve in the high-frequency region with the real axis represents the equivalent series resistance (ESR) of the electrodes. The CoFeCNTF electrode showed an ESR value of $2.38 \Omega$, smaller than that of Co-CNTF $(2.83 \Omega$ ). The smaller ESR and fast ion diffusion of $\mathrm{CoFe}-\mathrm{CNTF}$ electrode enhance the capacitive performance.
Additionally, to investigate the specific surface area and pore structure of CNTFs, $\mathrm{N}_{2}$ adsorption-desorption measurements were carried out. As shown in Figure 2D, both CoFe- and CoNi-CNTFs displayed type-IV isotherms with sharply increased adsorption at low pressures and distinct hysteresis loops at medium pressure region, indicating existence of micropores, and abundant mesopores (Ding et al., 2018). Compared with CoFeand CoNi-CNTFs, Co-CNTF exhibited a smaller hysteresis loop at high relative pressure, indicating that $\mathrm{CoFe}$ - and CoNi-CNTFs possesses more mesopores. Moreover, the pore size distributions of Co-CNTF, CoNi-CNTF, and CoFe-CNTF in Figure S5 also suggest more mesopores existed in $\mathrm{CoNi}-\mathrm{CNTF}$ and $\mathrm{CoFe}-\mathrm{CNTF}$ than that of Co-CNTF. The Brunauer-Emmett-Teller (BET) surface areas of CoFe and CoNi-CNTF are 410 and $369 \mathrm{~m}^{2} \mathrm{~g}^{-1}$, respectively, which is higher than that of Co-CNTF $\left(334 \mathrm{~m}^{2} \mathrm{~g}^{-1}\right)$. Obviously, the CoFe-CNTF with the highest specific surface area and abundant mesopore is in favor of the diffusion of ions, thus, it is promising electrode materials for CDI.

The CDI performance of the CoFe-CNTF electrode was further studied in terms of the effects of various applied voltages and concentrations of $\mathrm{NaCl}$ solution. As shown in Figure 3A, with the increase of the applied voltage, the electrosorption capacity was increased, indicating that a larger amount of ions were removed from the solution due to stronger electrostatic interaction. The corresponding transient current curves at different applied voltages in Figure 3B also show an increased current at higher voltages. Furthermore,

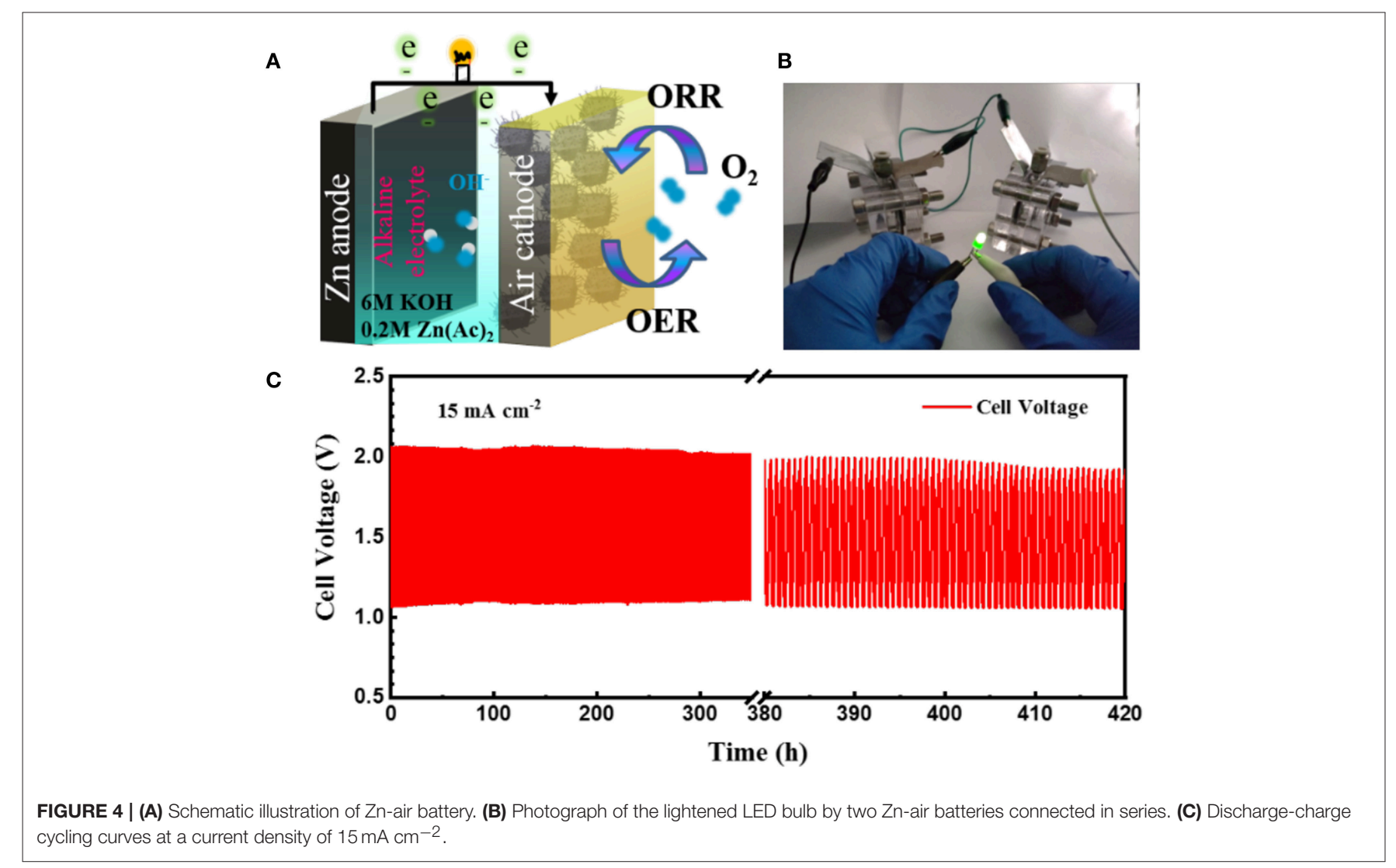


electrosorption experiments in $\mathrm{NaCl}$ solutions of various initial concentrations ranging from 125 to $1,000 \mathrm{mg} \mathrm{L}^{-1}$ were carried out. Figure 3C demonstrates the calculated adsorption capacities of CoFe-CNTF electrode in different concentrations of $\mathrm{NaCl}$ solution at cell voltages of $1.0,1.2,1.4$, and $1.6 \mathrm{~V}$, respectively. With increment of the concentration of $\mathrm{NaCl}$ solution, the adsorption capacity increases as well. Notably, the CoFe-CNTF electrode exhibited a superior adsorption capacity of $37.0 \mathrm{mg} \mathrm{g}^{-1}$ in a $500 \mathrm{mg} \mathrm{L}^{-1} \mathrm{NaCl}$ solution at $1.2 \mathrm{~V}$, which is superior to most of the recently reported CDI electrode materials (see Table S1). Furthermore, the regeneration stability of the electrode was investigated by repeated electrosorption-desorption experiments at a voltage of $1.2 \mathrm{~V}$. As shown in Figure 3D, CoFe-CNTF electrode exhibited excellent stability for 12 cycles without obvious capacity drop.

The above studies clearly demonstrate that CoFe-CNTF has an extraordinary specific surface area, conductivity, and electrochemical activity. This could be attributed to the hierarchically porous structure with abundant exposed active sites, high electrical conductivity for fast electron transfer, and unique nanostructures with $\mathrm{Co} / \mathrm{Fe}$ nanoparticles embedded in CNTFs. These excellent properties endow them promising performance as air electrode of $\mathrm{Zn}$-air batteries. As shown in Scheme 1, in order to apply it in flexible $\mathrm{Zn}$-air batteries, the OER performance of CoFe-CNTF was first investigated. The OER activity of the as-obtained CoFe-CNTF was evaluated by a conventional three-electrode cell in $1.0 \mathrm{M} \mathrm{KOH}$ solution. Commercial $\mathrm{IrO}_{2}$ was also tested for comparison. As shown in Figure S6A, the CoFe-CNTF catalyst require a potential of $1.574 \mathrm{~V}$ vs. RHE (corresponding to overpotential of $344 \mathrm{mV}$ ) to achieve a current density of $10 \mathrm{~mA} \mathrm{~cm}^{-2}$, which is better than that of commercial $\mathrm{IrO}_{2}(1.658 \mathrm{~V})$. Moreover, the Tafel slope of CoFe-CNTF is $87.7 \mathrm{mV} \mathrm{dec}^{-1}$, which is much smaller than that of commercial $\mathrm{IrO}_{2}\left(106.5 \mathrm{mV} \mathrm{dec}{ }^{-1}\right)$, suggesting faster OER catalytic kinetics of CoFe-CNTF (see Figure S6B). The Nyquist plots in Figure S6C demonstrate that the charge transfer resistance of $\mathrm{CoFe}-\mathrm{CNTF}$ is much smaller than that of commercial $\mathrm{IrO}_{2}$, indicating that charge transfer process within $\mathrm{CoFe}-\mathrm{CNTF}$ is more effective. Notably, CoFe-CNTF also exhibits excellent OER stability. As shown in Figure S6D, CoFe-CNTF shows nearly constant operating potential with a current density of $10 \mathrm{~mA} \mathrm{~cm}^{-2}$ for $12 \mathrm{~h}$.

To demonstrate the practical application of the obtained CoFe-CNTF, a rechargeable Zn-air battery was fabricated. Figure 4A shows a schematic diagram of the configuration of rechargeable Zn-air battery, in which CoFe-CNTF catalyst loaded on carbon paper was used as air-cathode, $\mathrm{Zn}$ plate as anode, and a mixed solution of $6 \mathrm{M} \mathrm{KOH}$ and $0.2 \mathrm{M} \mathrm{Zn}(\mathrm{Ac})_{2}$ as electrolyte. Two rechargeable $\mathrm{Zn}$-air batteries connected in series can light up a light-emitting diode (LED) bulb successfully (Figure 4B). Furthermore, the fabricated $\mathrm{Zn}$-air battery exhibited remarkable cycling stability after operation for $420 \mathrm{~h}$ at a current density of $15 \mathrm{~mA} \mathrm{~cm}^{-2}$ (Figure 4C). In addition, a flexible $\mathrm{Zn}$ air battery based on $\mathrm{CoFe}-\mathrm{CNTF}$ is also demonstrated. CoFeMOFs were grown on 3DG (see Figure S7) followed by a similar annealing process to produce the flexible air cathode
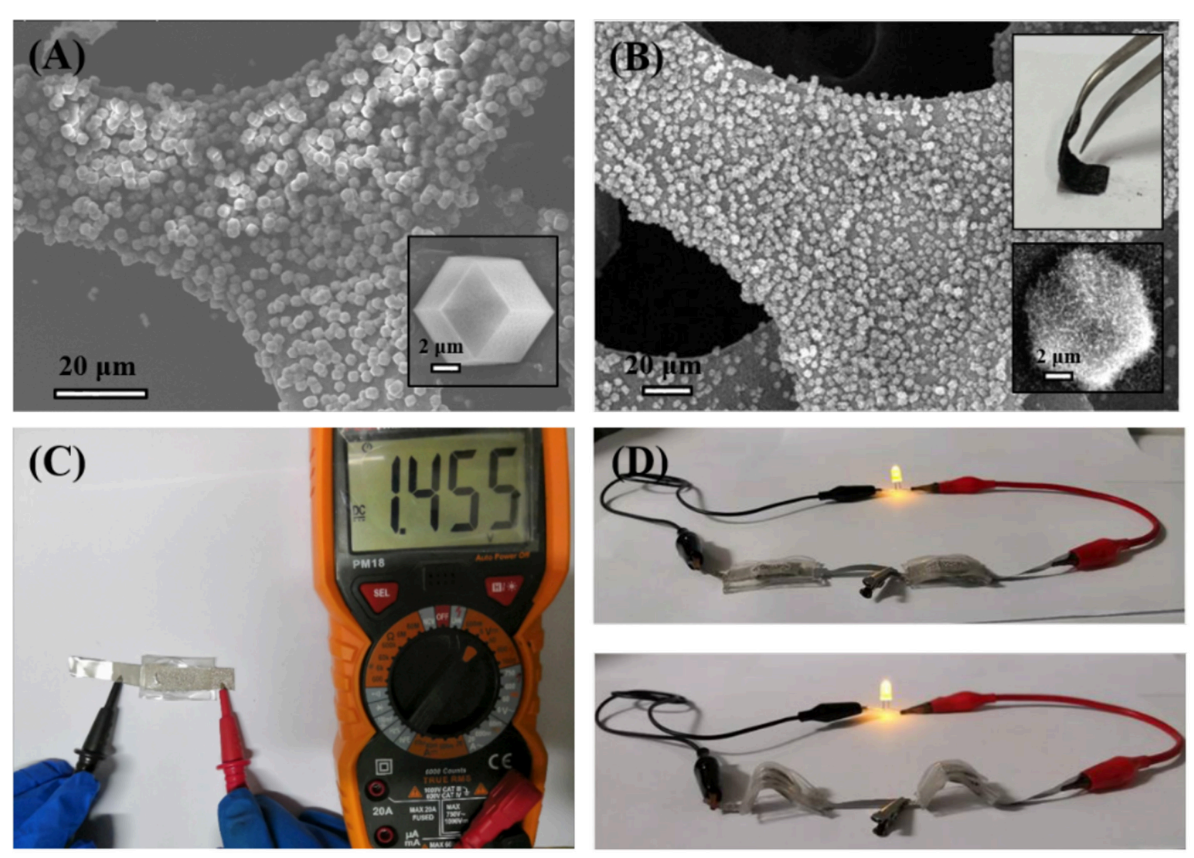

FIGURE 5 | (A) SEM image of CoFe-MOF@3DG. Inset is high-magnification SEM image showing a CoFe-MOF crystal grown on the surface of 3DG. (B) SEM image of CoFe-CNTF@3DG. Insets: photograph of a bent CoFe-CNTF@3DG, and high-magnification SEM image (Bottom). (C) Open-circuit voltage of a fabricated all-solid-state Zn-air battery based on CoFe-CNTF@3DG. (D) Photographs of the lightened LED by two Zn-air batteries connected in series under different blending status. 
of CoFe-CNTF@3DG. The morphologies and XRD patterns of obtained CoFe-MOF@3DG and CoFe-CNTF@3DG are shown in Figures 5A,B and Figure S8. The photograph in the top inset of Figure 5B clearly demonstrates good flexibility of the obtained CoFe-CNTF@3DG electrode. The open-circuit voltage of the fabricated all-solid-state Zn-air battery is $1.455 \mathrm{~V}$ (Figure 5C). Importantly, a LED bulb could be successfully lightened by two $\mathrm{Zn}$-air batteries connected in series under different blending status (Figure 5D), demonstrating its excellent flexibility and promising application in $\mathrm{Zn}$-air batteries.

\section{CONCLUSION}

In summary, we developed hierarchically porous carbon nanotube-based frameworks (CNTFs), which were derived from rationally designed bimetallic MOFs followed by a two-step annealing process. The CNTFs showed excellent $\mathrm{Na}^{+} / \mathrm{Cl}^{-}$ion adsorption property. Especially, the CoFe-CNTF exhibited a remarkable adsorption capacity of $37.0 \mathrm{mg} \mathrm{g}^{-1}$ in a $500 \mathrm{mg}$ $\mathrm{L}^{-1} \mathrm{NaCl}$ solution at $1.2 \mathrm{~V}$, superior to that of Co-CNTF (19.9 $\mathrm{mg} \mathrm{g}^{-1}$ ). Such enhanced performance can be attributed to the larger surface area and improved electrical conductivity of the CoFe-CNTF. Moreover, due to the unique porous framework structure and abundant metal active sites, the CoFe-CNTF displayed excellent electrocatalytic performance in OER.CoFe-CNTF@3DG as air electrode demonstrated excellent flexibility and potential application for flexible all-solid-state Zn-air battery. We believe our work paves a way for the design of nanostructured carbon materials for the applications in sustainable energy conversion and storage.

\section{REFERENCES}

Cao, X., Shi, Y., Shi, W., Lu, G., Huang, X., Yan, Q., et al. (2011). Preparation of novel 3D graphene networks for supercapacitor applications. Small 7, 3163-3168. doi: 10.1002/smll.201100990

Cao, X., Yin, Z., and Zhang, H. (2014). Three-dimensional graphene materials: preparation, structures and application in supercapacitors. Energy Environ. Sci. 7, 1850-1865. doi: 10.1039/C4EE00050A

Ding, M., Bannuru, K. K. R., Wang, Y., Guo, L., Baji, A., and Yang, H. Y. (2018). Free-standing electrodes derived from metal-organic frameworks/nanofibers hybrids for membrane capacitive deionization. Adv. Mater. Technol. 3:1800135. doi: 10.1002/admt.201800135

Ding, M., Shi, W., Guo, L., Leong, Z. Y., Baji, A., and Yang, H. Y. (2017). Bimetallic metal-organic framework derived porous carbon nanostructures for high performance membrane capacitive desalination. J. Mater. Chem. A 5, 6113-6121. doi: 10.1039/C7TA00339K

Fang, G., Wang, Q., Zhou, J., Lei, Y., Chen, Z., Wang, Z., et al. (2019). Metal organic framework-templated synthesis of bimetallic selenides with rich phase boundaries for sodium-ion storage and oxygen evolution reaction. ACS Nano. 13, 5635-5645. doi: 10.1021/acsnano.9b00816

Gao, T., Du, Y., and Li, H. (2019a). Preparation of nitrogen-doped graphitic porous carbon towards capacitive deionization with high adsorption capacity and rate capability. Sep. Purif. Technol. 211, 233-241. doi: 10.1016/j.seppur.2018.09.085

Gao, T., Li, H., Zhou, F., Gao, M., Liang, S., and Luo, M. (2019b). Mesoporous carbon derived from ZIF-8 for high efficient electrosorption. Desalination 451, 133-138. doi: 10.1016/j.desal.2017.06.021

Gao, T., Zhou, F., Ma, W., and Li, H. (2018). Metal-organic-framework derived carbon polyhedron and carbon nanotube hybrids as electrode for

\section{DATA AVAILABILITY}

All datasets generated for this study are included in the manuscript and/or the Supplementary Files.

\section{AUTHOR CONTRIBUTIONS}

WS, XC, JS, CG, and WL designed the project. WS, CY, XS, and XL performed the synthesis, CDI experiment, and data analysis. XX, DS, and RY performed the Zn-air battery experiment and data analysis. All authors contribute to editing and discussion of the manuscript.

\section{FUNDING}

This work was supported by the financial support from Zhejiang Provincial Natural Science Foundation of China (LQ17B030002, LR19E020003) and the National Natural Science Foundation of China (51702286, 51602284), General Scientific Research Project of the Department of Education of Zhejiang Province, China (Y201839638), and the Thousand Talent Program and Qianjiang Scholars program of Zhejiang Province in China.

\section{SUPPLEMENTARY MATERIAL}

The Supplementary Material for this article can be found online at: https://www.frontiersin.org/articles/10.3389/fchem. 2019.00449/full\#supplementary-material

electrochemical supercapacitor and capacitive deionization. Electrochim. Acta 263, 85-93. doi: 10.1016/j.electacta.2018.01.044

Guo, Q., Li, X., Wei, H., Liu, Y., Li, L., Yang, X., et al. (2019). Sr, Fe Co-doped perovskite oxides with high performance for oxygen evolution reaction. Front. Chem. 7:224. doi: 10.3389/fchem.2019.00224

Han, Y., Lu, Y., Shen, S., Zhong, Y., Liu, S., Xia, X., et al. (2018). Enhancing the capacitive storage performance of carbon fiber textile by surface and structural modulation for advanced flexible asymmetric supercapacitors. Adv. Mater. Funct. 29:1806329. doi: 10.1002/adfm.201806329

Hassanvand, A., Chen, G. Q., Webley, P. A., and Kentish, S. E. (2018). A comparison of multicomponent electrosorption in capacitive deionization and membrane capacitive deionization. Water Res. 131, 100-109. doi: 10.1016/j.watres.2017.12.015

Hu, C., Liu, Z., Lu, X., Sun, J., Liu, H., and Qu, J. (2018). Enhancement of the donnan effect through capacitive ion increase using an electroconductive rGO-CNT nanofiltration membrane. J. Mater. Chem. A 6, 4737-4745. doi: 10.1039/C7TA11003K

Huang, Z.-H., Yang, Z., Kang, F., and Inagaki, M. (2017). Carbon electrodes for capacitive deionization. J. Mater. Chem. A 5, 470-496. doi: 10.1039/C6TA06733F

Ji, D., Fan, L., Li, L., Peng, S., Yu, D., Song, J., et al. (2019). Atomically transition metals on self-supported porous carbon flake arrays as binderfree air-cathode for wearable zinc-air batteries. Adv. Mater. 31:e1808267. doi: 10.1002/adma.201808267

Kaur, G., Rai, R. K., Tyagi, D., Yao, X., Li, P.-Z., Yang, X.-C., et al. (2016). Room-temperature synthesis of bimetallic Co-Zn based zeolitic imidazolate frameworks in water for enhanced $\mathrm{CO}_{2}$ and $\mathrm{H}_{2}$ uptakes. J. Mater. Chem. A 4, 14932-14938. doi: 10.1039/C6TA04342A 
Lee, J., Jo, K., Lee, J., Hong, S. P., Kim, S., and Yoon, J. (2018). Rockingchair capacitive deionization for continuous brackish water desalination. ACS Sustain Chem. Eng. 6, 10815-10822. doi: 10.1021/acssuschemeng.8b0 2123

Li, H., Su, Y., Sun, W., and Wang, Y. (2016). Carbon nanotubes rooted in porous ternary metal sulfide@N/S-doped carbon dodecahedron: bimetalorganic-frameworks derivation and electrochemical application for highcapacity and long-life lithium-ion batteries. Adv. Funct. Mater. 26, 8345-8353. doi: 10.1002/adfm.201601631

Li, J., Ji, B., Jiang, R., Zhang, P., Chen, N., Zhang, G., et al. (2018a). Hierarchical hole-enhanced 3D graphene assembly for highly efficient capacitive deionization. Carbon 129, 95-103. doi: 10.1016/j.carbon.2017.1 1.095

Li, Y., Kim, J., Wang, J., Liu, N. L., Bando, Y., Alshehri, A. A., et al. (2018b). High performance capacitive deionization using modified ZIF-8-derived, Ndoped porous carbon with improved conductivity. Nanoscale 10, 14852-14859. doi: 10.1039/C8NR02288G

Liu, P., Yan, T., Shi, L., Park, H. S., Chen, X., Zhao, Z., et al. (2017a). Graphenebased materials for capacitive deionization. J. Mater. Chem. A, 5 13907-13943. doi: 10.1039/C7TA02653F

Liu, R., Sui, Y., and Wang, X. (2019a). Metal-organic framework-based ultrafltration membrane separation with capacitive-type for enhanced phosphate removal. Chem. Eng. J. 371, 903-913. doi: 10.1016/j.cej.2019.0 4.136

Liu, W., Huang, J., Yang, Q., Wang, S., Sun, X., Zhang, W., et al. (2017b). Multi-shelled hollow metal-organic frameworks. Angew. Chem. Int. Edit. 56, 5512-5516. doi: 10.1002/anie.2017 01604

Liu, W., Yin, R., Shi, W., Xu, X., Shen, X., Yin, Q., et al. (2018). Gram-scale preparation of $2 \mathrm{D}$ transition metal hydroxide/oxide assembled structures for oxygen evolution and Zn-air battery. ACS Appl. Energy Mater. 2, 579-586. doi: 10.1021 /acsaem.8b01613

Liu, W., Yin, R., Xu, X., Zhang, L., Shi, W., and Cao, X. (2019b). Structural engineering of low-dimensional metal-organic frameworks: synthesis, properties, and applications. Adv. Sci. 1802373. doi: 10.1002/advs.2018 02373

Liu, Y., Lu, T., Sun, Z., Chua, D. H. C., and Pan, L. (2015). Ultra-thin carbon nanofiber networks derived from bacterial cellulose for capacitive deionization. J. Mater. Chem. A 3, 8693-8700. doi: 10.1039/C5TA00 435G

Lv, Z., Tang, Y., Zhu, Z., Wei, J., Li, W., Xia, H., et al. (2018). Honeycomblantern-inspired 3D stretchable supercapacitors with enhanced specific areal capacitance. Adv. Mater. 30:1805468. doi: 10.1002/adma.201805468

Nie, C., Pan, L., Liu, Y., Li, H., Chen, T., Lu, T., et al. (2012). Electrophoretic deposition of carbon nanotubes-polyacrylic acid composite film electrode for capacitive deionization. Electrochim. Acta 66, 106-109. doi: 10.1016/j.electacta.2012.01.064

Peng, S., Han, X., Li, L., Chou, S., Ji, D., Huang, H., et al. (2018). Electronic and defective engineering of electrospun $\mathrm{CaMnO}_{3}$ nanotubes for enhanced oxygen electrocatalysis in rechargeable zinc-air batteries. Adv. Energy Mater. 8:1800612. doi: 10.1002/aenm.201800612

Shi, W., Li, H., Cao, X., Leong, Z. Y., Zhang, J., Chen, T., et al. (2016). Ultrahigh performance of novel capacitive deionization electrodes based on a three-dimensional graphene architecture with nanopores. Sci. Rep. 6:18966. doi: 10.1038/srep 18966

Shi, W., Liu, X., Ye, C., Cao, X., Gao, C., and Shen, J. (2019a). Efficient lithium extraction by membrane capacitive deionization incorporated with monovalent selective cation exchange membrane. Sep. Purif. Technol. 210, 885-890. doi: 10.1016/j.seppur.2018. 09.006

Shi, W., Mao, J., Xu, X., Liu, W., Zhang, L., Cao, X., et al. (2019b). An ultra-dense NiS2/reduced graphene oxide composite cathode for highvolumetric/gravimetric energy density nickel-zinc batteries. J. Mater. Chem. A. doi: 10.1039/c9ta04900b. [Epub ahead of print].

Shi, W., Ye, C., Xu, X., Liu, X., Ding, M., Liu, W., et al. (2018). Highperformance membrane capacitive deionization based on metal-organic framework-derived hierarchical carbon structures. ACS Omega 3, 8506-8513. doi: 10.1021/acsomega.8b01356
Song, J., Ma, J., Zhang, C., He, C., and Waite, D. (2019). Implication of nonelectrostatic contribution to deionization in flow-electrode CDI: case study of nitrate removal from contaminated source waters. Front. Chem. 7:146. doi: $10.3389 /$ fchem. 2019.00146

Suss, M. E., Porada, S., Sun, X., Biesheuvel, P. M., Yoon, J., and Presser, V. (2015). Water desalination via capacitive deionization: what is it and what can we expect from it? Energy Environ. Sci. 8, 2296-2319. doi: 10.1039/C5EE0 0519A

Tang, J., Salunkhe, R. R., Liu, J., Torad, N. L., Imura, M., Furukawa, S., et al. (2015). Thermal conversion of core-shell metal-organic frameworks: a new method for selectively functionalized nanoporous hybrid carbon. J. Am. Chem. Soc. 137, 1572-1580. doi: 10.1021/ja511539a

Tang, W., Liang, J., He, D., Gong, J., Tang, L., Liu, Z., et al. (2019). Various cell architectures of capacitive deionization: recent advances and future trends. Water Res. 150, 225-251. doi: 10.1016/j.watres.2018.1 1.064

Tsouris, C., Mayes, R., Kiggans, J., Sharma, K., Yiacoumi, S., DePaoli, D., et al. (2011). Mesoporous carbon for capacitive deionization of saline water. Environ. Sci. Technol. 45, 10243-10249. doi: 10.1021/es201 $551 \mathrm{e}$

Wang, H., Shi, L., Yan, T., Zhang, J., Zhong, Q., and Zhang, D. (2014). Design of graphene-coated hollow mesoporous carbon spheres as high performance electrodes for capacitive deionization. J. Mater. Chem. A 2, 4739-4750. doi: 10.1039/C3TA15152B

Wang, M., Xu, X., Liu, Y., Li, Y., Lu, T., and Pan, L. (2016). From metalorganic frameworks to porous carbons: a promising strategy to prepare high-performance electrode materials for capacitive deionization. Carbon 108, 433-439. doi: 10.1016/j.carbon.2016.07.047

Wang, M., Xu, X., Tang, J., Hou, S., Hossain, M. S. A., Pan, L., et al. (2017a). High performance capacitive deionization electrodes based on ultrathin nitrogendoped carbon/graphene nano-sandwiches. Chem. Commun. 53, 10784-10787. doi: 10.1039/C7CC05673G

Wang, Z., Xu, X., Kim, J., Malgras, V., Mo, R., Li, C., et al. (2019). Nanoarchitectured metal-organic framework/polypyrrole hybrids for brackish water desalination using capacitive deionization. Mater. Horiz. doi: 10.1039/C9MH00306A. [Epub ahead of print].

Wang, Z., Yan, T., Shi, L., and Zhang, D. (2017b). In situ expanding pores of dodecahedron-like carbon frameworks derived from MOFs for enhanced capacitive deionization. ACS Appl. Mater. Interfaces 9, 15068-15078. doi: 10.1021/acsami.7b02712

Wu, T., Wang, G., Zhan, F., Dong, Q., Ren, Q., Wang, J., et al. (2016) Surface-treated carbon electrodes with modified potential of zero charge for capacitive deionization. Water Res. 93, 30-37 doi: 10.1016/j.watres.2016.0 2.004

Xu, X., Allah, A., Wang, C., Tan, H., Farghali, A., Khedr, M., et al. (2019). Capacitive deionization using nitrogen-doped mesostructured carbons for highly efficient brackish water desalination. Chem. Eng. J. 362, 887-896. doi: 10.1016/j.cej.2019.01.098

Xu, X., Li, J., Wang, M., Liu, Y., Lu, T., and Pan, L. (2016a). Shuttlelike porous carbon rods from carbonized metal-organic frameworks for high-performance capacitive deionization. Chemelectrochem 3, 993-998. doi: 10.1002/celc.201600051

Xu, X., Shi, W., Li, P., Ye, S., Ye, C., Ye, H., et al. (2017). Facile fabrication of three-dimensional graphene and metal-organic framework composites and their derivatives for flexible all-solid-state supercapacitors. Chem. Mater. 29, 6058-6065. doi: 10.1021/acs.chemmater.7b01947

Xu, X., Shi, W., Liu, W., Ye, S., Yin, R., Zhang, L., et al. (2018). Preparation of two-dimensional assembled NiMn-C ternary composites for high-performance all-solid-state flexible supercapacitors. J. Mater. Chem. A 6, 24086-24091. doi: 10.1039/C8TA06412A

Xu, X., Wang, M., Liu, Y., Lu, T., and Pan, L. (2016b). Metal-organic frameworkengaged formation of a hierarchical hybrid with carbon nanotube inserted porous carbon polyhedra for highly efficient capacitive deionization. J. Mater. Chem. A 4, 5467-5473. doi: 10.1039/C6TA00618C

Zhang, L., Liu, W., Shi, W., Xu, X., Mao, J., Li, P., et al. (2018a). Boosting lithium storage properties of MOF derivatives through a wet-spinning assembled fiber strategy. Chem-Eur. J. 24, 13792-13799. doi: 10.1002/chem.20180 2826 
Zhang, W., Ma, X., Zhong, C., Ma, T., Deng, Y., Hu, W., et al. (2018b). Pyrite-type $\mathrm{CoS}_{2}$ nanoparticles supported on nitrogen-doped graphene for enhanced water splitting. Front. Chem. 6:569. doi: 10.3389/fchem.2018.00569

Zheng, X., Cao, Y., Liu, D., Cai, M., Ding, J., Liu, X., et al. (2019). Bimetallic metal-organic-framework/reduced graphene oxide composites as bifunctional electrocatalysts for rechargeable Zn-air batteries. ACS Appl. Mater. Interfaces 11, 15662-15669. doi: 10.1021/acsami.9b02859

Zhu, L., Zheng, D., Wang, Z., Zheng, X., Fang, P., Zhu, J., et al. (2018). A confinement strategy for stabilizing ZIF-derived bifunctional catalysts as a benchmark cathode of flexible all-solid-state zinc-air batteries. Adv. Mater. 30:1805268. doi: 10.1002/adma.201805268
Conflict of Interest Statement: The authors declare that the research was conducted in the absence of any commercial or financial relationships that could be construed as a potential conflict of interest.

Copyright (C) 2019 Shi, Xu, Ye, Sha, Yin, Shen, Liu, Liu, Shen, Cao and Gao. This is an open-access article distributed under the terms of the Creative Commons Attribution License (CC BY). The use, distribution or reproduction in other forums is permitted, provided the original author(s) and the copyright owner(s) are credited and that the original publication in this journal is cited, in accordance with accepted academic practice. No use, distribution or reproduction is permitted which does not comply with these terms. 